\title{
High Stability White Organic Light-Emitting Diode (WOLED) Using Nano-Double-Ultra Thin Carrier Trapping Materials
}

\author{
Kan-Lin Chen \\ Department of Electronic Engineering, Fortune Institute of Technology, Kaohsiung, Taiwan \\ Correspondence should be addressed to Kan-Lin Chen; klchen@fotech.edu.tw
}

Received 25 January 2014; Accepted 28 January 2014; Published 6 March 2014

Academic Editor: Chien-Jung Huang

Copyright ( 2014 Kan-Lin Chen. This is an open access article distributed under the Creative Commons Attribution License, which permits unrestricted use, distribution, and reproduction in any medium, provided the original work is properly cited.

\begin{abstract}
The structure of indium tin oxide (ITO) $(100 \mathrm{~nm}) /$ molybdenum trioxide $\left(\mathrm{MoO}_{3}\right)(15 \mathrm{~nm}) / \mathrm{N}, \mathrm{N} 0$-bis-(1-naphthyl)-N,N0biphenyl-1,10-biphenyl-4,40-diamine (NPB) (40 nm)/4,4'-Bis(2,2-diphenylvinyl)-1,1'-biphenyl (DPVBi) (10 nm)/5,6,11,12tetraphenylnaphthacene (Rubrene) $(0.2 \mathrm{~nm}) / \mathrm{DPVBi}(24 \mathrm{~nm}) /$ Rubrene $(0.2 \mathrm{~nm}) / \mathrm{DPVBi}(6 \mathrm{~nm}) / 4,7$-diphenyl-1,10-phenanthroline (BPhen): cesium carbonate $\left(\mathrm{Cs}_{2} \mathrm{Co}_{3}\right)(10 \mathrm{~nm}) / \mathrm{Al}(120 \mathrm{~nm})$ with high color purity and stability white organic light-emitting diode (WOLED) was fabricated. The function of the multiple-ultra-thin material (MUTM), such as Rubrene, is as the yellow light-emitting layer and trapping layer. The results show that the MUTM has an excellent carrier capture effect, resulting in high color stability of the device at different applied voltages. The Commissions Internationale De L'Eclairage (CIE) coordinate of this device at $3 \sim 7 \mathrm{~V}$ is few displacement and shows a very slight variation of $( \pm 0.01, \pm 0.01)$. The maximum brightness of $9986 \mathrm{~cd} / \mathrm{m}^{2}$ and CIE coordinates of $(0.346,0.339)$ are obtained at $7 \mathrm{~V}$. The enhanced performance of the device may result from the direct charge trapping in MUTM and it can be found in the electroluminescence (EL) process.
\end{abstract}

\section{Introduction}

Recently, organic light-emitting diodes (OLED) have attracted a great deal of attention as the new full-color displays and solid-state lighting technology. OLED's superior characteristics such as high brightness, fast response time, wide viewing angle, and low operating voltage allow it to have great potential for commercialization [1-3]. Furthermore, the white organic light-emitting diode (WOLED) lighting technology has been extensively studied. And then, there are several methods to obtain WOLEDs; for example, using a multilayer stack of three primary colors (red, green, and blue), two complementary colors (blue and yellow), and two or three colors of the dye doped into a single host material [4-7]. In the doping codeposition process, to accurately control the evaporation rate and the concentration of two or more materials is very difficult, resulting in its deposition process and reproducibility being poor. However, nondoping technique can accurately control to avoid these problems in the fabrication process. Currently, Tsuji et al. and Xie et al. have reported non-doped-type WOLEDs with the single-ultra-thin layer (SUTL) structure, which is a simple device structure with good reproducibility, making them very suitable for low-cost lighting applications and conducive to commercialization $[8,9]$.

In this work, we employ the nondoped method to fabricate WOLEDs with a multiple-ultra-thin material (MUTM) structure. The material of 5-, 6-, 11-, 12-tetraphenylnaphthacene (Rubrene) was used as yellow light sources in the MUTM structure. Rubrene is the most common nanomaterial, which has good light-emitting efficiency, high color saturation, good stability, and good capture efficiency of charge carriers. In addition, the photoluminescence (PL) quantum efficiency of the Rubrene can be close to $100 \%$ [10-13]. And then the material of 4, 40-bis (2, 20-diphenyl vinyl)-1, 10biphenyl (DPVBi) is a well-known blue fluorescent material. It is usually deemed a host-emitting layer (EML) due to the higher efficiency of blue light. Moreover, DPVBi not only emits but also transfers the incomplete energy from DPVBi to the Rubrene. So far the study of fluorescence WOLED based on the nondoped MUTM is rarely reported. Therefore, we introduced a simple process for the nondoped WOLEDs with an MUTM structure. Meanwhile, the influential factors for the improvement of WOLEDs performance were investigated 
in detail. However, the mechanism of the influences of an MUTM structure on the electroluminescence (EL) and the optimal color stability of WOLEDs is also presented.

\section{Experimental Details}

Indium tin oxide (ITO) coated glass with a sheet resistance of approximately $12 \Omega$ /sq was consecutively cleaned in an ultrasonic bath containing detergent water, acetone, ethanol, and deionized (DI) water for 20 min each, then dried with a nitrogen $\left(\mathrm{N}_{2}\right)$ flow. All organic nanomaterials were deposited by high-vacuum $\left(10^{-6}\right.$ Torr) thermal evaporation. Thermal deposition rates for organic nanomaterials, inorganic materials, and $\mathrm{Al}$ were about $0.1 \AA / \mathrm{sec}, 1 \AA / \mathrm{sec}$, and $10 \AA / \mathrm{sec}$, respectively. The evaporation rate and thickness of the thin films were monitored using a quartz crystal oscillator system (Sigma, SID-142). The structures of devices are as follows:

(A) ITO/molybdenum trioxide $\left(\mathrm{MoO}_{3} ; 15 \mathrm{~nm}\right) / \mathrm{N}, \mathrm{N} 0$ bis-(1-naphthyl)-N,N0-biphenyl-1,10-biphenyl-4,40diamine (NPB; $40 \mathrm{~nm}) / \mathrm{DPVBi} \quad(10 \mathrm{~nm}) /$ Rubrene (0.2 nm)/DPVBi $(30 \mathrm{~nm}) / 4,7-$ diphenyl-1,10-phenanthroline: cesium carbonate (BPhen: $\mathrm{Cs}_{2} \mathrm{Co}_{3}=4: 1$; $10 \mathrm{~nm}$ )/aluminum (Al; $120 \mathrm{~nm})$,

(B) $\mathrm{ITO} / \mathrm{MoO}_{3}(15 \mathrm{~nm}) / \mathrm{NPB}(40 \mathrm{~nm}) / \mathrm{DPVBi}(34 \mathrm{~nm}) /$ Rubrene $(0.2 \mathrm{~nm}) / \mathrm{DPVBi}(6 \mathrm{~nm}) / \mathrm{BPhen}: \mathrm{Cs}_{2} \mathrm{Co}_{3}=$ $4: 1(10 \mathrm{~nm}) / \mathrm{Al}(120 \mathrm{~nm})$,

(C) $\mathrm{ITO} / \mathrm{MoO}_{3}(15 \mathrm{~nm}) / \mathrm{NPB}(40 \mathrm{~nm}) / \mathrm{DPVBi}(10 \mathrm{~nm}) /$ Rubrene (0.2 nm)/DPVBi $(24 \mathrm{~nm}) /$ Rubrene $(0.2 \mathrm{~nm}) /$ DPVBi $(6 \mathrm{~nm}) /$ BPhen: $\mathrm{Cs}_{2} \mathrm{Co}_{3}=4: 1(10 \mathrm{~nm}) / \mathrm{Al}$ $(120 \mathrm{~nm})$.

In these devices, $\mathrm{MoO}_{3}$ and NPB are used as a holeinjecting layer and hole-transport layer. The DPVBi acts as a blue-emitting layer. The ultrathin Rubrene (UTR) was selected as a yellow light-emitting layer. The BPhen: $\mathrm{Cs}_{2} \mathrm{Co}_{3}$ is used as an electron transporting layer and hole blocking layer. The chemical structures of the organic nanomaterials and the structures of the device are shown in Figure 1. The active area of the device was $0.4 \mathrm{~cm}^{2}$. With measuring the properties of the device, a voltage was applied by using a Keithley 2400 programmable voltage-current source (Keithley SourceMeter 2400; USA). EL spectra and CIE coordination of the devices were measured by PR655 spectra scan spectrometer (Kollmorgen Instrument PR655; USA). All measurements were carried out at room temperature in air without encapsulating the devices.

\section{Results and Discussion}

In this study, the WOLED which consists of the blue emission layer and the yellow emission layer was fabricated. The UTR layer was inserted in the light-emitting layer of DPVBi to form the structure of DPVBi (10 nm EML1)/Rubrene $(0.2 \mathrm{~nm}) / \mathrm{DPVBi}(30 \mathrm{~nm}$ EML2) for device A. The yellow emission of device A was caused by the UTR layer. Figure 2(a) shows the EL spectra of device $A$ at the applied voltage of $3 \sim 7 \mathrm{~V}$. The peak wavelengths of the DPVBi layer and the Rubrene layer were $436 \mathrm{~nm}$ and $556 \mathrm{~nm}$, respectively [14, 15]. The intensity of blue emission was higher than that of the yellow emission, and the location of EL spectra for the blue and the yellow emission did not shift as the voltage increased.

In addition, the CIE coordinates of device $\mathrm{A}$ at the applied voltage of 3 7 V were shown in Figure 2(b). It was found that the CIE coordinates for device A changed from (0.269, $0.299)$ at $3 \mathrm{~V}$ to $(0.249,0.259)$ at $7 \mathrm{~V}$. By comparing the CIE coordinates $(0.330,0.330)$ of standard white light, the error value of the CIE coordinates was about $(-0.081,-0.071)$ at the $7 \mathrm{~V}$; that is, the shift of the CIE coordinates is about $(-7.4 \%$, $-13.4 \%$ ) during the applied voltage of $3 \sim 7 \mathrm{~V}$. That is to say, the CIE coordinates of device A were unstable. As for the EL phenomenon in the applied voltage of $3 \sim 5 \mathrm{~V}$, some of the holes which were injected from the anode via Rubrene layer into the EML2 can be directly trapped by the UTR layer, which might be due to the effective hole trapping of Rubrene molecules. However, the UTR layer has excellent charge carriers trapping properties, but it is unable to trap lots of the injected holes. On the other hand, the electron mobility in BPhen: $\mathrm{Cs}_{2} \mathrm{Co}_{3}$ of $3.9 \times 10^{-4} \mathrm{~cm}^{2} / \mathrm{Vs}$ was less than the hole mobility of $5.5 \times 10^{-4} \mathrm{~cm}^{2} / \mathrm{Vs}$ in NPB, so lots of the electrons from an electrode inject just into EML2; that is, the electrons recombine with holes in the EML2 close cathode. Therefore, the electrons were difficult to be directly trapped by the UTR layer at 3 5 V. As a result, the majority of electrons and holes would meet in EML2. Similarly, for the voltage of $6 \sim 7 \mathrm{~V}$, more charge carriers were injected into Rubrene layer and EML2, so the amount of recombination for electrons and holes was also relatively increased in Rubrene layer and EML2, resulting in an enhancement in the intensity of peaks, as shown in Figure 2(a). However, the UTR layer in device A cannot trap enough electrons and holes to generate exciton. Therefore, optimum complementary color of the blue and the yellow emission intensity was not achieved.

According to the results obtained above, the best recombination zone was in the EML2 of device A. For device B, the UTR layer inserted in and closed to the cathode to form the structure of DPVBi (34 nm EML1)/Rubrene (0.2 nm)/DPVBi (6 nm EML2) was fabricated. However, by comparing Figures 2(a) and 3(a), it is found that the yellow emission intensity of device $B$ was stronger than that of device A because of its position in the UTR layer being changed. Although the UTR layer was inserted into the best recombination zone and has an excellent charge carrier trapping effect, the blue emission and the yellow emission did not reach the best complementary color.

As mentioned above, the optimal location is in EML2 of device A. Thus, the UTR layer was inserted into a better recombination zone near the EML2/BPhen: $\mathrm{Cs}_{2} \mathrm{Co}_{3}$ interface of device $\mathrm{B}$. The difference between lowest unoccupied molecular orbital (LUMO) energy of Rubrene $(-3.2 \mathrm{eV})$ and that of DPVBi $(-2.8 \mathrm{eV})$ is $0.4 \mathrm{eV}$. Similarly, the difference between highest occupied molecular orbital (HOMO) value of Rubrene $(-5.4 \mathrm{eV})$ and that of DPVBi $(-5.9 \mathrm{eV})$ is $0.5 \mathrm{eV}$ $[16,17]$. It is expected that the electrons and holes can be trapped in the UTR layer as well as accumulated in the Rubrene/EML2 interface, leading to an enhancement in 


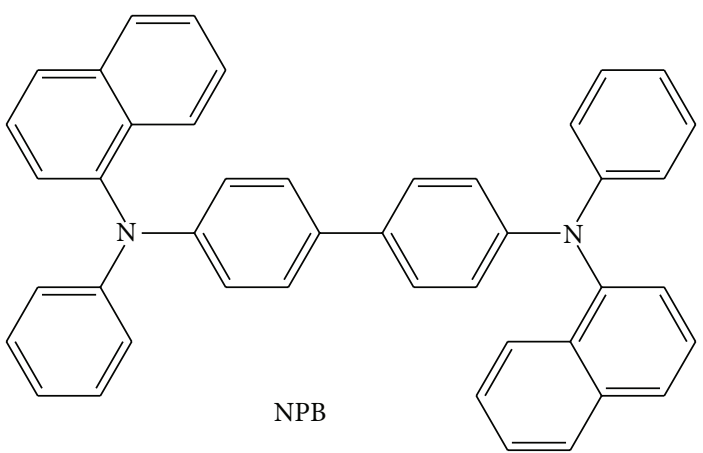

(a)<smiles>c1ccc(-c2c3ccccc3c(-c3ccccc3)c3c(-c4ccccc4)c4ccccc4c(-c4ccccc4)c23)cc1</smiles>

Rubrene

(c)

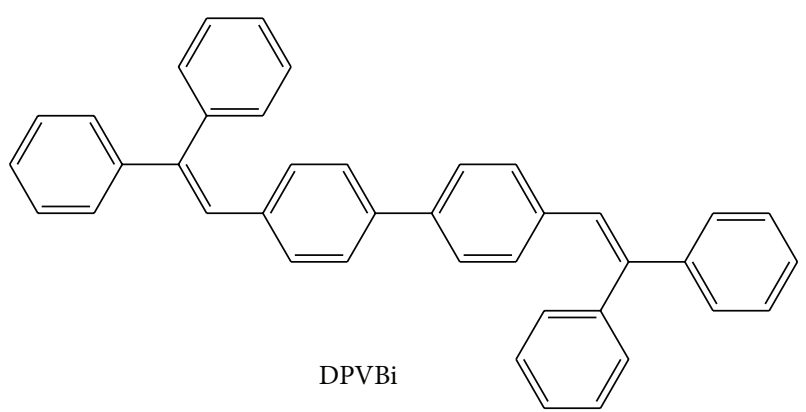

(b)

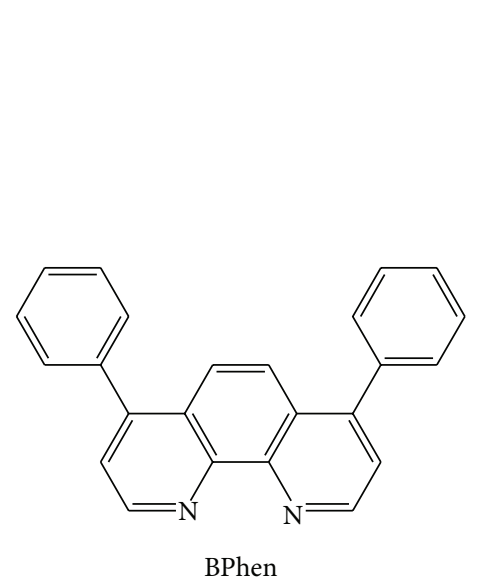

(d)

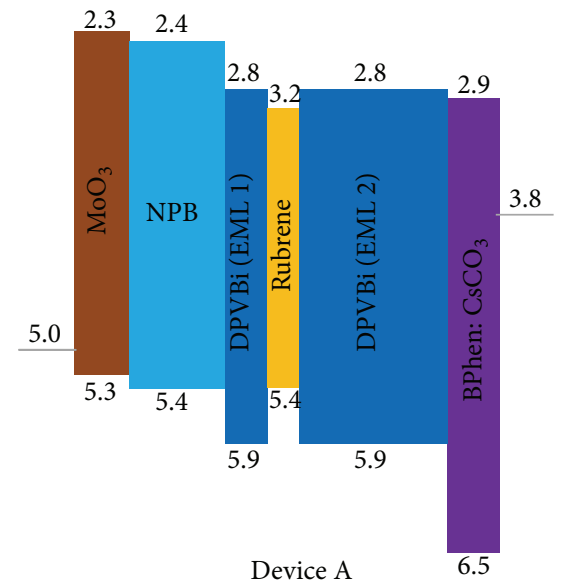

(e)

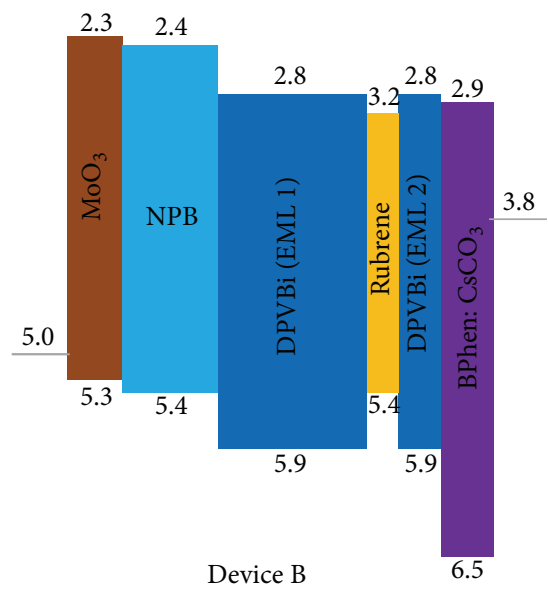

(f)

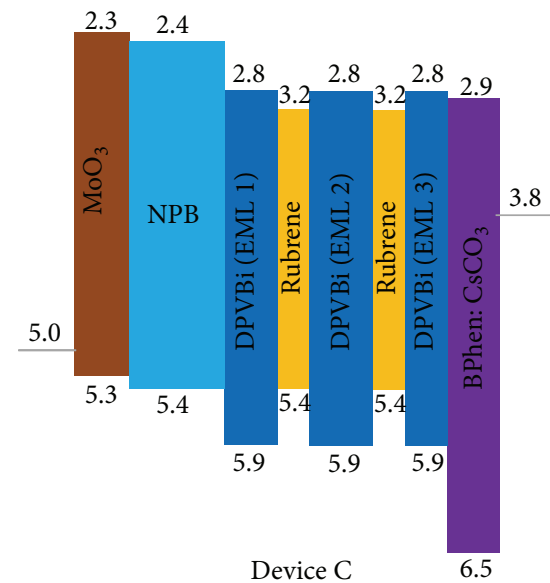

(g)

Figure 1: The chemical structures of (a) NPB, (b) DPVBi, (c) Rubrene, and (d) BPhen and the schematic energy band diagrams of (e) device $A$, (f) device B, and (g) device C.

the intensity of yellow emission. Figure 3 shows the EL spectra and CIE coordinates of device B. With the applied voltage increasing, most of the electrons and holes can be directly trapped and recombined to form exciton in the UTR layer. Moreover, the relative intensity of yellow is higher than that of the blue emission; that is, the exciton number of UTR layer is more than that of the DPVBi layer. In Figure 3(a), the intensity of both yellow emission and blue emission became large, but the ratio of the enhancement in yellow emission is larger than that of blue emission. Thus, the CIE coordinate gradually shifts toward an orientation of white color. For example, when the applied voltage is $3 \mathrm{~V}$ and $4 \mathrm{~V}$, the location of CIE coordinates shows at $(0.462$, $0.481)$ and $(0.425,0.437)$, respectively. By comparing the CIE coordinates of standard white light, the error value of the CIE coordinates was gradually reduced. It is confirmed that the 


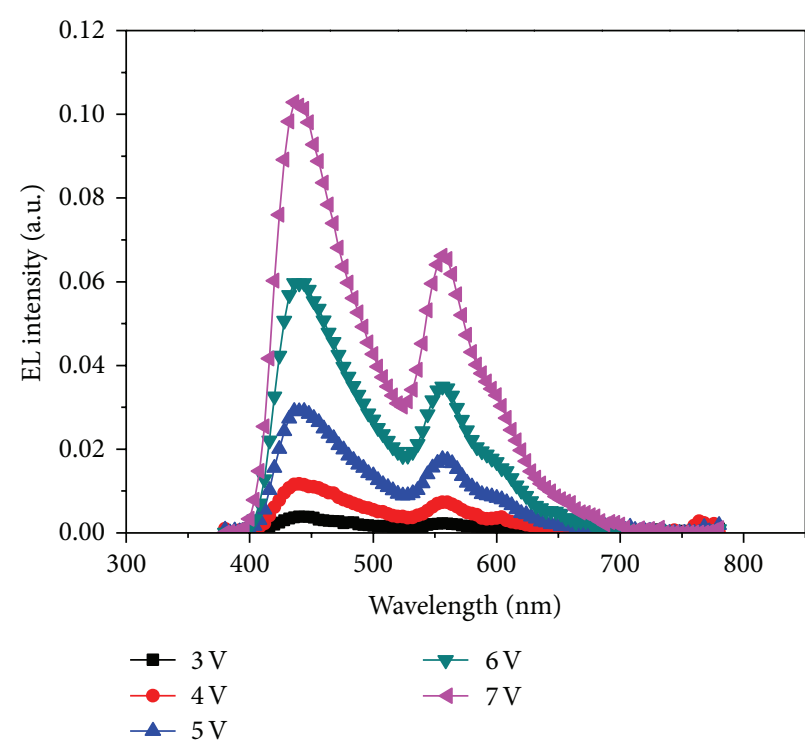

(a)

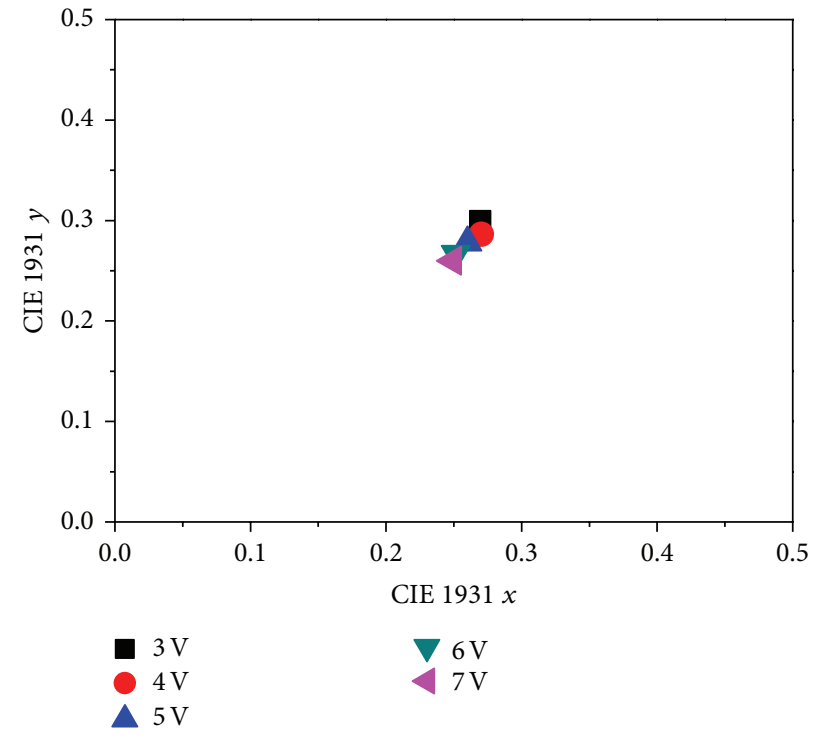

(b)

Figure 2: (a) The EL spectra of device A at 3 7 V applied voltage. (b) The CIE coordinates of device A at 3 7 V applied voltage.

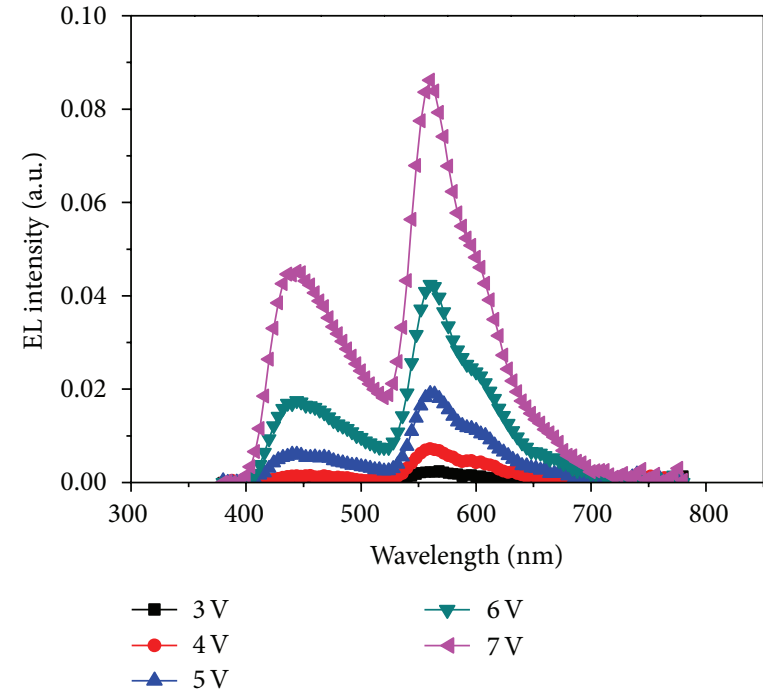

(a)

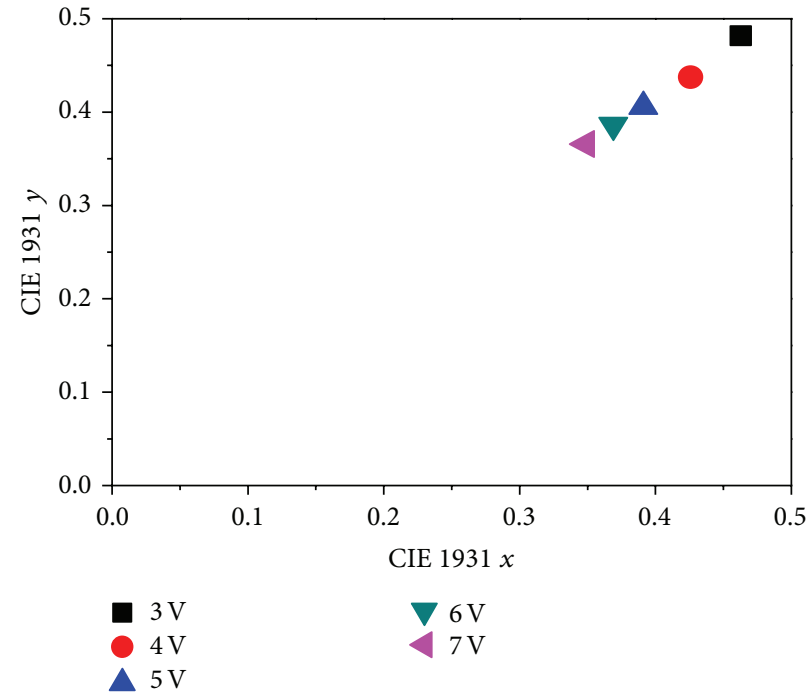

(b)

Figure 3: (a) The EL spectra of device B at 3 7 V applied voltage. (b) The CIE coordinates of device B at 3 7 V applied voltage.

better recombination zone of device B is in the UTR/EML2 interface.

With the applied voltage increasing to $5 \mathrm{~V}$, the recombination zone of electrons and holes gradually shifts toward the UTR/EML2 interface; the corresponding CIE coordinates are $(0.391,0.405)$. We thus conjecture that few excitons are generated in the EML2, resulting in an enhancement in the blue emission. On the other hand, more electrons and holes were trapped in the UTR; that is, more excitons are generated in the UTR, leading to an enhanced yellow emission. At the voltage of $6 \sim 7 \mathrm{~V}$, CIE coordinates shift again from $(0.368,0.385)$ to $(0.348,0.365)$ and gradually shifts toward the CIE coordinates of standard white light. When a high voltage is applied, the concentration of electrons and holes increases which significantly influences the zone of the exciton generation. The movement of the electrons and holes is similar towards the opposite electrode. Thus, the zone of the exciton generation in device $\mathrm{B}$ becomes broad as the EML1/UTR and UTR/EML2 interfaces. This implies that the location of UTR not only improves the color shift of CIE coordinates but also enhances carrier recombination rate. By comparing the CIE coordinates of standard white light, the error value of the CIE coordinates was about $(0.018,0.035)$ at the $7 \mathrm{~V}$; that is, the shift of the CIE coordinates is about 


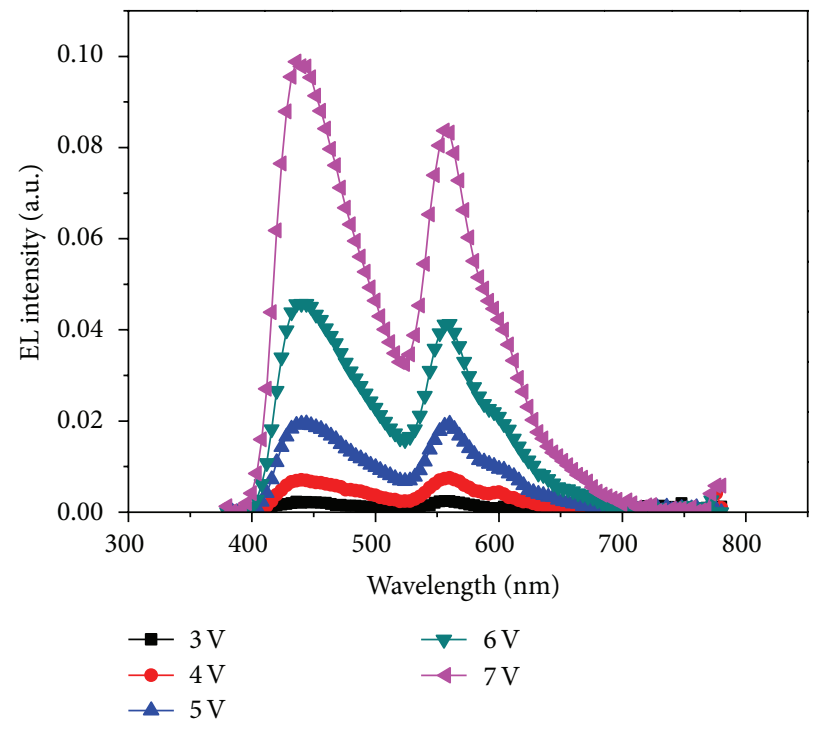

(a)

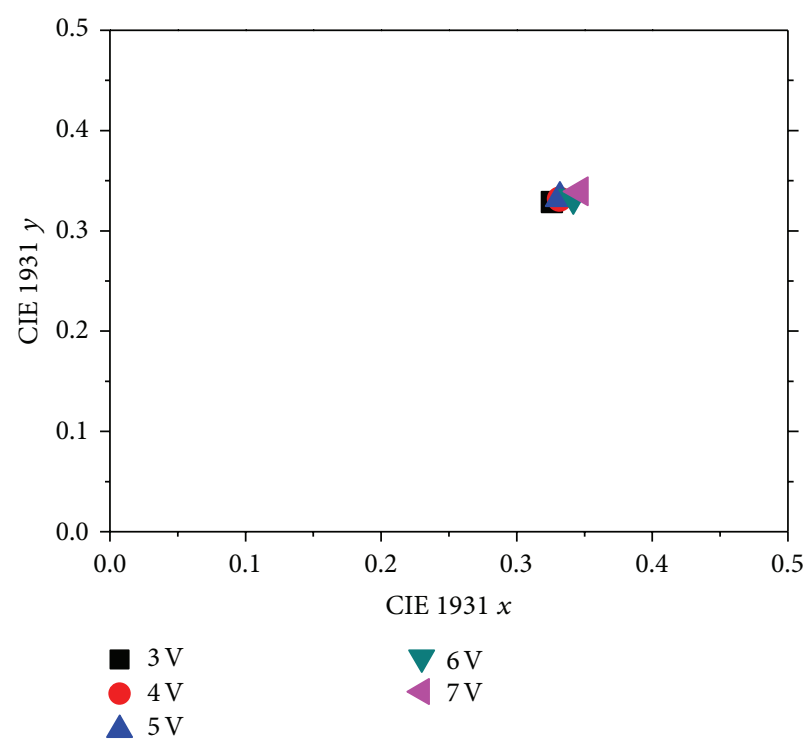

(b)

FIgure 4: (a) The EL spectra of device $\mathrm{C}$ at 3 7 V applied voltage. (b) The CIE coordinates of device $\mathrm{C}$ at 3 7 V applied voltage.

$(-24.7 \%,-24.1 \%)$ during the applied voltage of $3 \sim 7 \mathrm{~V}$. With the results obtained above, the CIE coordinates of device B are close to the CIE coordinates of standard white light by the ultrathin Rubrene layer inserted into EML2, but the CIE coordinates of device $\mathrm{B}$ are still unstable during the applied voltage of $3 \sim 7 \mathrm{~V}$.

Figure 4 shows the EL spectra and CIE coordinates of device C, ITO/ $\mathrm{MoO}_{3} / \mathrm{NPB} / \mathrm{DPVBi} / \mathrm{Rubrene} / \mathrm{DPVBi} /$ Rubrene/DPVBi/BPhen: $\mathrm{Cs}_{2} \mathrm{Co}_{3} / \mathrm{Al}$, during the applied voltage of $3 \sim 7 \mathrm{~V}$. It is found that there is a balance or complementary color in blue emission and yellow emission, as shown in Figure 4(a). Besides, at the applied voltage of $5 \mathrm{~V}$, a pure white emission with CIE coordinates of $(0.332,0.332)$ is observed. When the voltage continued to increase to 6 and $7 \mathrm{~V}$, the CIE coordinates were, respectively, $(0.342,0332)$ and $(0.345,0.338)$, and showed little change. This is due to the fact that the ratio of blue and yellow emission intensity from EL spectra of Figure 4(a) is almost the same and about unit. Furthermore, this improvement in chromaticity can be attributed to the MUTM structure in the emission layer, resulting in a balance in the relative intensity of blue and yellow emission. Thus, by introducing an MUTM structure in the emission layer, the WOLED has more stable spectra characteristics than that of devices A and B with the increase of bias voltage. This is due to the reason that the injection of holes and electrons was enhanced by the MUTM structure and that the MUTM structure can enhance charge carrier trapping in the EML1/Rubrene, EML2/Rubrene, and Rubrene/EML3 interfaces. And then the enhanced carrier injection was because electric fields were induced by the accumulated carrier at the MUTM. This result is similar to the result discussed in other literatures [18-21]. In other words, the excitons formed from electrons and holes can widely distribute in the entire emission layer. As is expected, the excitons of Rubrene and DPVBi layers will increase and reach balance, indicating that the MUTM can enhance color stability of CIE coordinates and chromaticity of pure white emission. However, the difference of the CIE coordinates during the applied voltage of $3 \sim 7 \mathrm{~V}$ is almost null which is advantageous for lighting application.

The current density-voltage-luminance characteristics of devices $\mathrm{A} \sim \mathrm{C}$ are shown in Figure 5. The maximum current density of devices $\mathrm{A}, \mathrm{B}$, and $\mathrm{C}$ is $204 \mathrm{~mA} / \mathrm{cm}^{2}, 298 \mathrm{~mA} / \mathrm{cm}^{2}$, and $356 \mathrm{~mA} / \mathrm{cm}^{2}$ at $7 \mathrm{~V}$, respectively. It can be seen that device $\mathrm{C}$ shows larger current density than the other two devices at the same voltage. This is the reason why it has larger luminance. The maximum luminance of devices A$\mathrm{C}$ is $6013 \mathrm{~cd} / \mathrm{m}^{2}, 7769 \mathrm{~cd} / \mathrm{m}^{2}$, and $9986 \mathrm{~cd} / \mathrm{m}^{2}$, respectively. It can be explained that the holes were accumulated at the Rubrene/EML3 interface and that the electrons were accumulated at the BPhen: $\mathrm{Cs}_{2} \mathrm{Co}_{3}$ layer, indicating that the electric field can be formed in EML3. The electric field is favorable for electron injection into the emission layer. However, it is expected that the holes were trapped and confined in the Rubrene/EML3 interface and electron injection thus enhanced, resulting in that an improved carrier balance was achieved. Moreover, when the MUTM structure was inserted into the emission layer, the current density rising in device $\mathrm{C}$ exceeds those in the other devices. The phenomenon can be attributed to the effect of Coulomb attractive force (CAF) that is due to the presence of the trapped and accumulated holes in the emission layer [22]. That is to say, more accumulated holes in the HOMO level of DPVBi/Rubrene/DPVBi potential well will generate the stronger CAF for electron, resulting in a better injection of electron from ETL to EML layer. Thus, the current density rising depends on the position and the number of the ultrathin layer, that is, the formation of MUTM.

Figure 6 shows the current efficiency and power efficiency of the device with and without MUTM. In Figure 6(a), the 


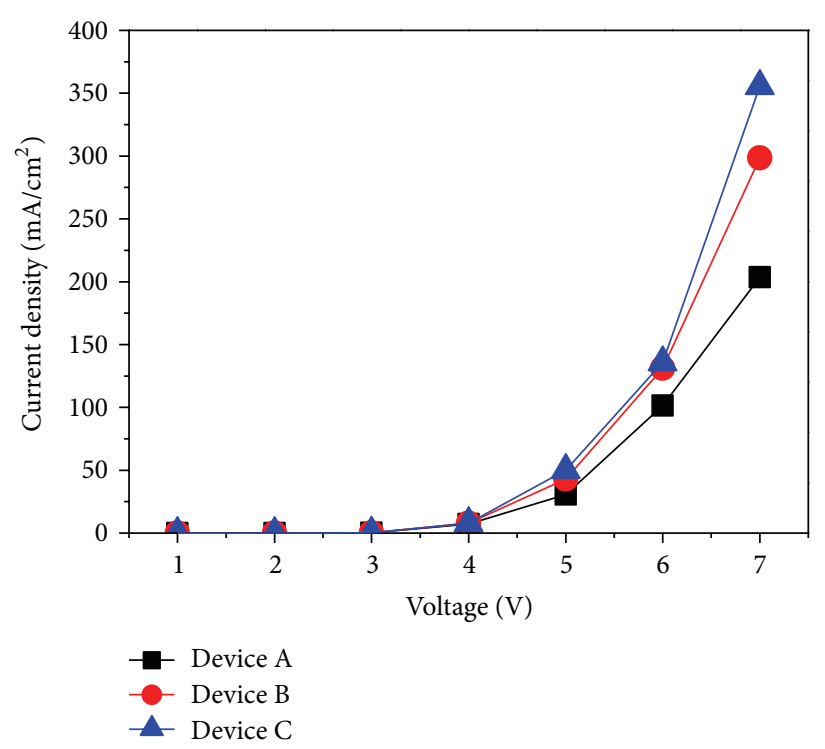

(a)

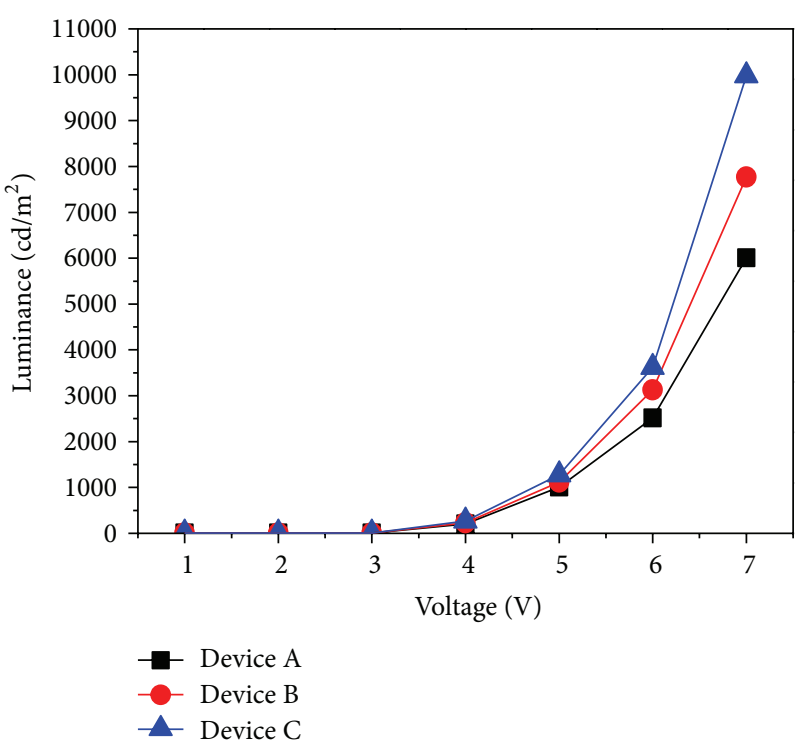

(b)

FIGURE 5: (a) The current density versus bias voltage (J-V) characteristics of devices A C. (b) The luminance versus bias voltage (L-V) characteristics of devices $\mathrm{A} \sim \mathrm{C}$.

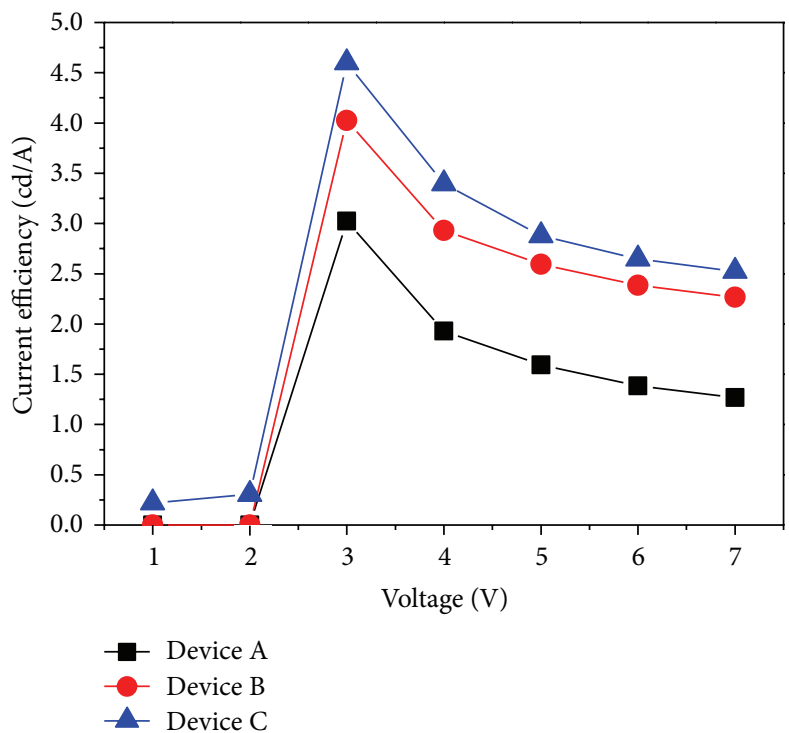

(a)

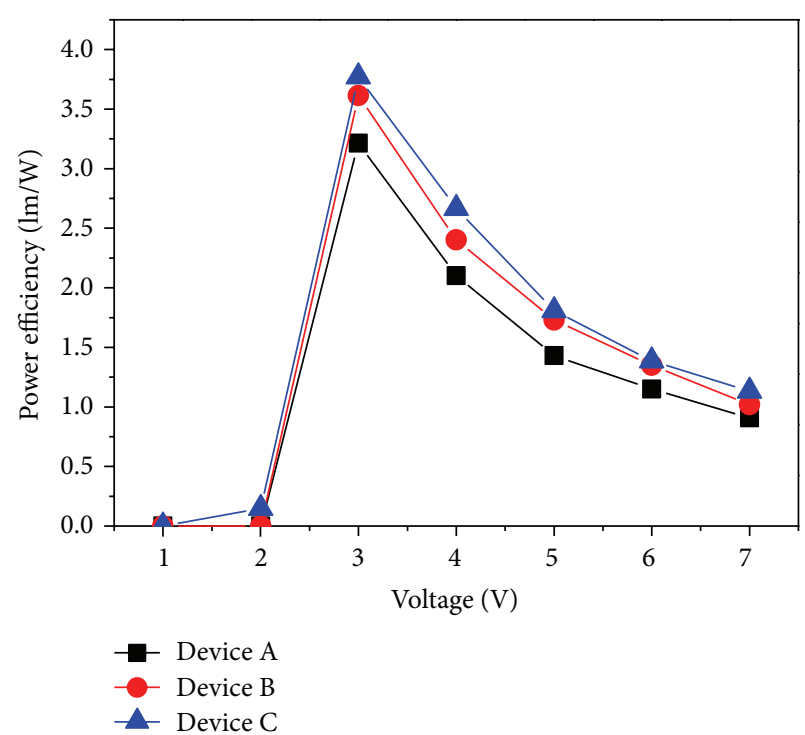

(b)

Figure 6: (a) The current efficiency versus bias voltage characteristics of devices A C. (b) The power efficiency versus bias voltage characteristics of devices A C.

current efficiency of device C with MUTM is of good and exceeds that without MUTM. At the applied voltage of $3 \mathrm{~V}$, devices $\mathrm{A}, \mathrm{B}$, and $\mathrm{C}$ show the maximum current efficiency of $3.02,4.02$, and $4.6 \mathrm{~cd} / \mathrm{A}$, respectively. These values indicate that the recombination rate of electron-hole pairs in device $\mathrm{C}$ exceed those in the other devices. In Figure 6(b), the maximum power efficiency of devices $\mathrm{A}-\mathrm{C}$ is 3.2, 3.6, and $3.7 \mathrm{~lm} / \mathrm{W}$, respectively. It can be seen that device $\mathrm{C}$ with the MUTM structure has a high power efficiency than the other two devices. As is known, the power efficiency depends on carrier injection, while current efficiency not only depends on the carrier injection, but also on the carrier balance. On the contrary, an unbalanced injection of electron-hole pairs in the EML with excess carrier injection reduces the efficiency due to the effect of carrier quench. Thus, the high current efficiency and power efficiency of the device can be achieved at an efficient carrier balance. However, the MUTM structure with high carrier trapped efficiency leads to the formation of stronger CAF, which enhances the efficiency of the device. 


\section{Conclusions}

In summary, white light emission was achieved by inserting the MUTM structure in the emission layer. The carrier trapping mechanism of the MUTM structure can more effectively confine electrons and holes in the emission layer. Consequently, the excitons of Rubrene and DPVBi layers will increase and reach balance; that is, there is a balance or complementary color in blue emission and yellow emission, resulting in good color stability characteristics of WOLED at different applied voltages. Pure white emission CIE coordinates of $(0.332,0.332)$ can be obtained and show little change at the applied voltage of $3 \sim 7 \mathrm{~V}$. It is demonstrated that EL emission is less dependent on the applied voltage. Furthermore, a device with a MUTM structure increased carrier trapping probability to improve carrier recombination, current density, and luminance intensity.

\section{Conflict of Interests}

The authors declare that there is no conflict of interests regarding the publication of this paper.

\section{Acknowledgments}

The author thanks the Research Center for Electro-Optical and Semiconductor, Southern Taiwan University of Technology, Taiwan, for fabrication and measurements of WOLED. The author would also like to thank Dr. Chien-Jung Huang and Mr. Fong-Yi for their discussion and support.

\section{References}

[1] C. J. Huang, T. H. Meen, S. L. Wu, and C. C. Kang, "Improvement of color purity and electrical characteristics by co-doping method for flexible red-light organic light emitting devices," Displays, vol. 30, no. 4-5, pp. 164-169, 2009.

[2] D. W. Chou, K. L. Chen, C. J. Huang, W. R. Chen, and T. H. Meen, "Effect of post-annealing treatment on silicon dioxide films for passivating flexible organic light-emitting diode," Solid-State Electronics, vol. 79, pp. 130-132, 2013.

[3] C. J. Huang, C. C. Kang, T. C. Lee, W. R. Chen, and T. H. Meen, "Improving the color purity and efficiency of blue organic light-emitting diodes (BOLED) by adding hole-blocking layer," Journal of Luminescence, vol. 129, no. 11, pp. 1292-1297, 2009.

[4] J. Kido, H. Shionoya, and K. Nagai, "Single-layer white lightemitting organic electroluminescent devices based on dyedispersed poly(N-vinylcarbazole)," Applied Physics Letters, vol. 67, no. 16, pp. 2281-2283, 1995.

[5] K. Okumoto, H. Kanno, Y. Hamada, H. Takahashi, and K. Shibata, "High efficiency red organic light-emitting devices using tetraphenyldibenzoperiflanthene-doped rubrene as an emitting layer," Applied Physics Letters, vol. 89, no. 1, Article ID 013502, 3 pages, 2006.

[6] G. Li and J. Shinar, "Combinatorial fabrication and studies of bright white organic light-emitting devices based on emission from rubrene-doped $4,4^{\prime}$-bis $\left(2,2^{\prime}\right.$-diphenylvinyl)-1,1' biphenyl," Applied Physics Letters, vol. 83, no. 26, pp. 5359-5361, 2003.

[7] K. T. Kamtekar, A. P. Monkman, and M. R. Bryce, "Recent advances in white organic light-emitting materials and devices
(WOLEDS)," Advanced Materials, vol. 22, no. 5, pp. 572-582, 2010.

[8] T. Tsuji, S. Naka, H. Okada, and H. Onnagawa, "Nondoped-type white organic electroluminescent devices utilizing complementary color and exciton diffusion," Applied Physics Letters, vol. 81, no. 18, pp. 3329-3331, 2002.

[9] W. F. Xie, Z. J. Wu, S. Y. Liu, and S. T. Lee, "Non-doped-type white organic light-emitting devices based on yellow-emitting ultrathin 5,6,11,12-tetraphenylnaphthacene and blue-emitting 4,4' - bis(2,2' -diphenyl vinyl)-1,1' - biphenyl," Journal of Physics D, vol. 36, no. 19, pp. 2331-2334, 2003.

[10] H. Mattoussi, H. Murata, C. D. Merritt, Y. Iizumi, J. Kido, and Z. H. Kafafi, "Photoluminescence quantum yield of pure and molecularly doped organic solid films," Journal of Applied Physics, vol. 86, no. 5, pp. 2642-2650, 1999.

[11] Y. M. Wang, F. Teng, Z. Xu, Y. B. Hou, S. Y. Yang, and X. R. $\mathrm{Xu}$, "Trap effect of an ultrathin DCJTB layer in organic lightemitting diodes," Materials Chemistry and Physics, vol. 92, no. 2-3, pp. 291-294, 2005.

[12] M. Matsumura and T. Furukawa, "Efficient electroluminescence from a rubrene sub-monolayer inserted between electron- and hole-transport layers," Japanese Journal of Applied Physics, vol. 40, no. 5, pp. 3211-3214, 2001.

[13] T. Li, X. Li, W. Li et al., "Tunable red emission by incorporation of a rubrene derivative in $p$-type and $n$-type hosts in organic light emitting devices," Thin Solid Films, vol. 517, no. 16, pp. 4629-4632, 2009.

[14] C.-J. Huang, T.-H. Meen, K.-C. Liao, and Y.-K. Su, "The mechanism of efficiency enhancement with proper thickness of DPVBi layer for blue organic light-emitting devices (BOLED)," Journal of Physics and Chemistry of Solids, vol. 70, no. 3-4, pp. 765-768, 2009.

[15] Q. Xue, S. Zhang, G. Xie et al., "Efficient fluorescent white organic light-emitting devices based on a ultrathin $5,6,11,12$ tetraphenylnaphthacene layer," Solid-State Electronics, vol. 57, no. 1, pp. 35-38, 2011.

[16] D. Xu, X. Li, H. Ju, Y. Zhu, and Z. Deng, "A novel red organic light-emitting diode with ultrathin DCJTB and Rubrene layers," Displays, vol. 32, no. 2, pp. 92-95, 2011.

[17] X. Y. Zheng, W. Q. Zhu, Y. Z. Wu et al., "A white OLED based on DPVBi blue light emitting host and DCJTB red dopant," Displays, vol. 24, no. 3, pp. 121-124, 2003.

[18] L. Li, J. Yu, X. Tang, T. Wang, W. Li, and Y. Jiang, "Efficient bright white organic light-emitting diode based on non-doped ultrathin 5,6,11,12-tetraphenylnaphthacene layer," Journal of Luminescence, vol. 128, no. 11, pp. 1783-1786, 2008.

[19] X. Tang, J. Yu, L. Li, L. Zhang, and Y. Jiang, "White organic lightemitting diodes with improved performance using phosphorescent sensitizer and ultrathin fluorescent emitter," Displays, vol. 30, no. 3, pp. 123-127, 2009.

[20] S. H. Yang, B. C. Hong, and S. F. Huang, "Luminescence enhancement and emission color adjustment of white organic light-emitting diodes with quantum-well-like structures," Journal of Applied Physics, vol. 105, no. 11, Article ID 113105, 7 pages, 2009.

[21] Z. Ma, J. Yu, L. Li, and Y. Jiang, "Non-doped white organic lightemitting diodes consisting of three primary colors based on a bipolar emitter," Displays, vol. 33, no. 1, pp. 42-45, 2012.

[22] Q. Xu, J. Ouyang, Y. Yang, T. Ito, and J. Kido, "Ultrahigh efficiency green polymer light-emitting diodes by nanoscale interface modification," Applied Physics Letters, vol. 83, no. 23, pp. 4695-4697, 2003. 

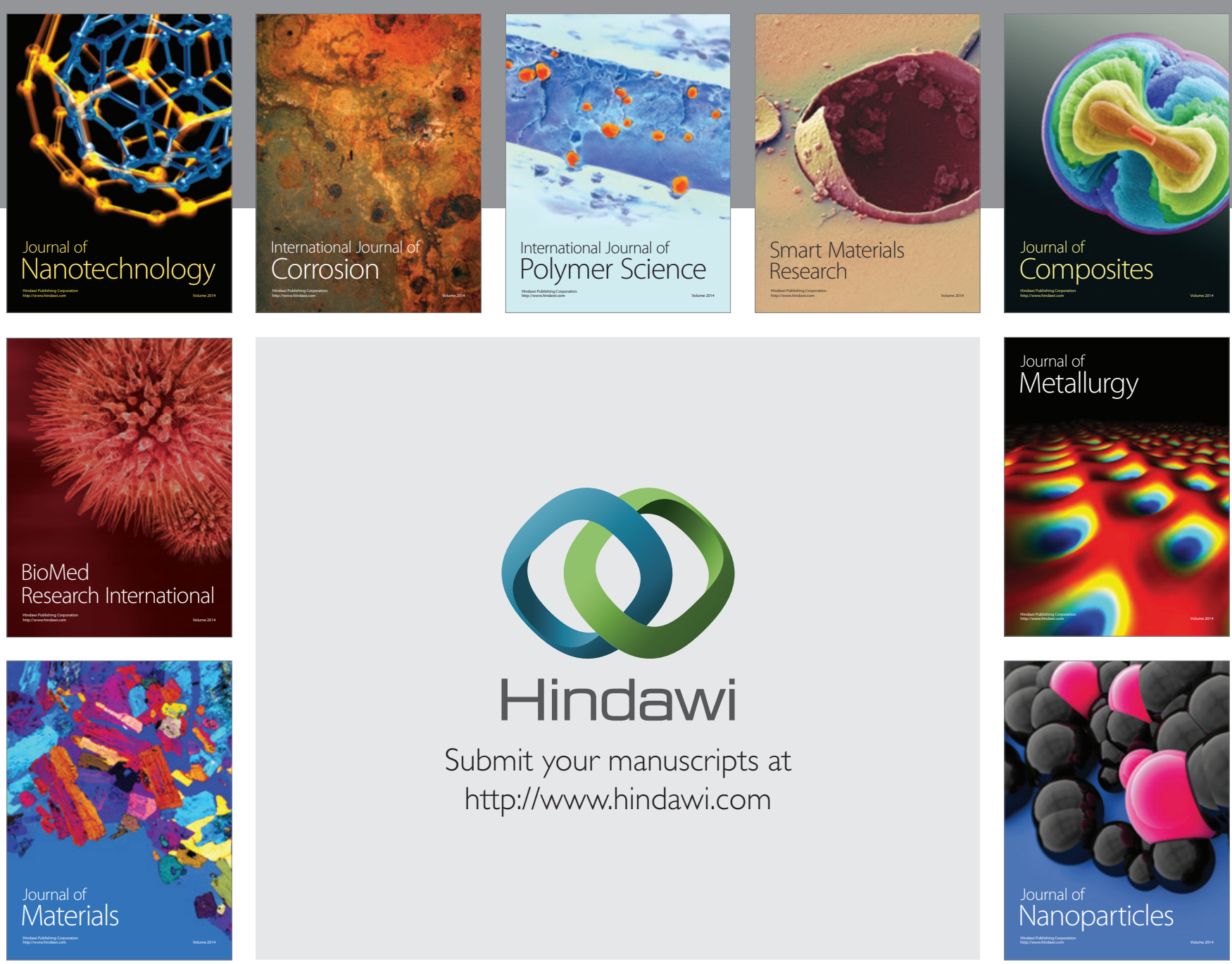

Submit your manuscripts at http://www.hindawi.com
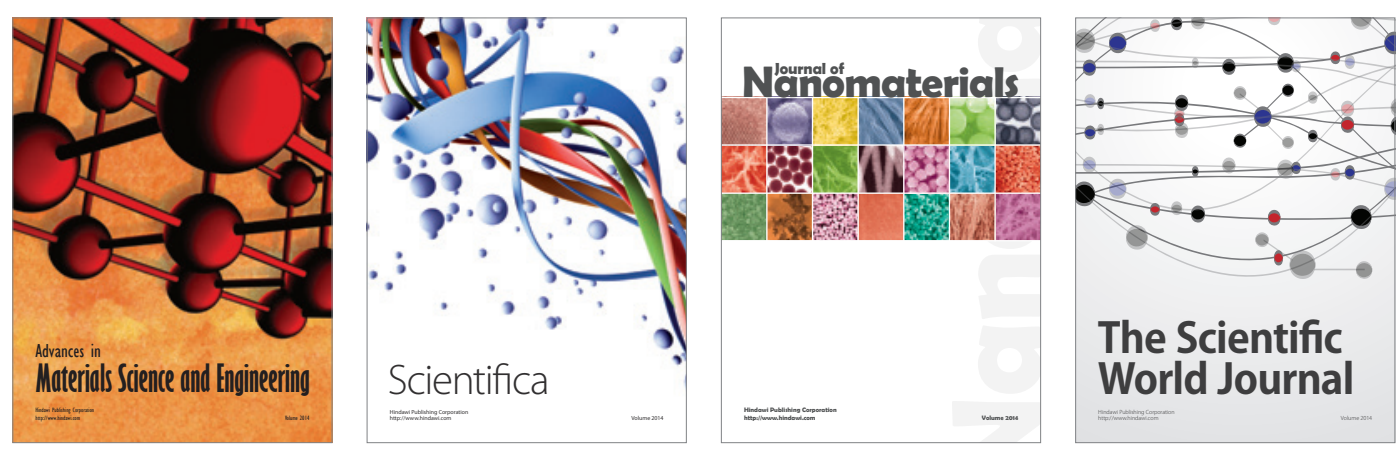

\section{The Scientific World Journal}
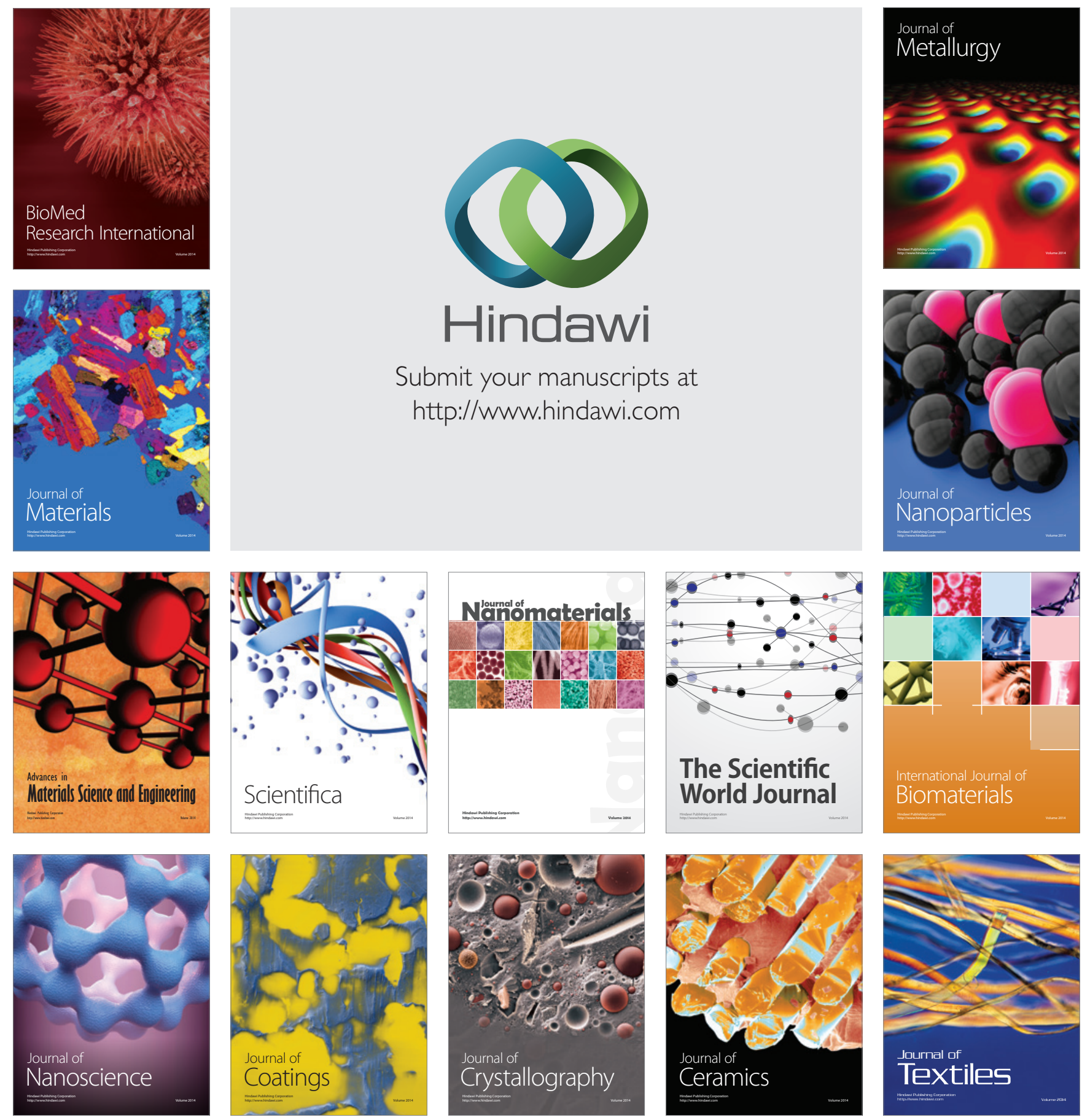